\title{
Evolução da qualidade de vida de cuidadores de pacientes com distrofia muscular de Duchenne
}

\section{Evolution of the quality of life of caregivers of patients with Duchenne muscular dystrophy}

Lorrane Caroline de Oliveira, Iriana Moraes Eduardo, Thaísa Fernandes Souza, Lorena Gomes de Medeiros, Cejane Oliveira Martins Prudente, Maysa Ferreira Martins Ribeiro

\section{RESUMO}

Objetivo: Analisar a evolução da qualidade de vida de cuidadores de pacientes com Distrofia Muscular de Duchenne (DMD) após um ano de reabilitação, segundo o grau de estadiamento da doença. Métodos: Estudo quantitativo e longitudinal, realizado em uma instituição de reabilitação de Goiânia - Goiás. A amostra foi composta por 27 pacientes com DMD e seus cuidadores familiares. Os instrumentos aplicados foram: ficha de perfil sociodemográfico; Escala Vignos; e Medida de Qualidade de Vida da Organização Mundial de Saúde (WHOQOL- bref). Resultados: Média de idade dos cuidadores foi de 39,44 anos e dos pacientes de 14,59 anos, a média da Escala de Vignos foi de 7,59 pontos. A Escala de

Como citar este artigo:

Oliveira, LC; Eduardo, IM; Souza, TF; Medeiros, LG; Prudente, COM; Ribeiro, MFM; Evolução da qualidade de vida de cuidadores de pacientes com distrofia muscular de Duchenne. Revista Saúde (Sta. Maria). 2018; 44 (3).

\section{Autor correspondente:}

Nome: Lorrane Caroline de

Oliveira

E-mail: lorrane_carol.

oliveira@hotmailcom

Telefone: ( 64 ) 996716115

Formação Profissional:

Graduanda em Fisioterapia pela

Universidade Estadual de Goiás

(UEG), Goiânia, CO, Brasil.

Filiação Institucional: Universidade

Estadual de Goiás

Endereço para correspondência:

Rua: $402 \quad n^{\circ}: 26 \quad$ Bairro:

Negrão de Lima Cidade:

Coiânia Estado: CO CEP:

74650340

Data de Submissão:

29/07/2018

Data de aceite:

22/01/2019

Conflito de Interesse: Não há conflito de interesse
Vignos apresentou piora significativa após um ano $(p=<0,001)$. Com relação ao WHOQOL-bref, o domínio meio ambiente mostrou as piores médias nas duas coletas, e o domínio físico as melhores. Não houve diferença significativa nas médias dos domínios e escore geral do WHOQOL-bref após um ano. Houve piora nos domínios psicológico e social apenas nos cuidadores cujos filhos eram mais graves. Conclusão: Cuidadores de pacientes mais graves, que encontram-se em fase de estadiamento da doença mais avançada, necessitam de um cuidado multiprofissional diferenciado, pois foram os que apresentaram pior qualidade de vida após um ano de acompanhamento.

Descritores: Cuidadores; Distrofia Muscular de Duchenne; Qualidade de vida;

\section{ABSTRACT}

Objective: to analyze the evolution of the quality of life of caregivers of patients with Duchenne Muscular Dystrophy (DMD) after a year of rehabilitation, according to the degree of disease. Methods: quantitative and longitudinal Study, conducted in a rehabilitation institution in Goiânia-Goiás. The sample was composed of 27 patients with DMD and their family caregivers. The instruments were applied: demographic profile; Vignos Scale; and measure of quality of life of the World Health Organization (WHOQOL-bref). Results: mean age of carers was 39.44 years and patients of 14.59 years, the average scale of Vignos was 7.59 points. Vignos scale showed significant worsening after one year $(p=<0.001)$. With respect to the WHOQOL-bref, the domain environment showed the worst averages in the two collections, and the physical domain. There was no significant difference in the averages of the domains and WHOQOL-bref overall score after one year. There were decreases in psychological and social domains only caregivers whose children were more severe. Conclusion: Caregivers of patients more graves, which are in a phase of more advanced disease, require multidisciplinary care, because they were the ones who presented a worse quality of life after one year of follow-up.

Descriptors: Caregivers; Duchenne Muscular Dystrophy; Quality of life; 


\section{INTRODUÇÃO}

A Distrofia Muscular de Duchenne (DMD) é uma das doenças neuromusculares geneticamente mais devastadoras ${ }^{1}$. Está associada à degeneração muscular progressiva e a necessidade de cuidados prologados ${ }^{2}$. Afeta 1 em 3500 recém-nascidos do sexo masculino ${ }^{3}$.

A DMD manifesta-se precocemente na infância. Os sinais clínicos são evidentes entre os três e cinco anos, quando as crianças apresentam dificuldade em sentar-se, levantar-se, e as quedas são frequentes ${ }^{4}$ devido à fraqueza muscular. A evolução dos sintomas é generalizada e irreversível, de forma simétrica e ascendente, incapacitando a deambulação entre nove e 13 anos $^{5}$. Os pacientes ficam confinados a cadeira de rodas após a perda da marcha, 0 que agrava as deformidades de coluna e membros inferiores, interferindo na independência funcional e autoestima ${ }^{6}$.

Nas últimas décadas a melhora nas condições de tratamento tem possibilitado maior expectativa de vida a esses pacientes, sendo a sobrevida marcada até a terceira década de vida ${ }^{7}$. Contudo, por ainda ser uma doença incurável o impacto nos aspectos psicossociais desses pacientes e da família pode influenciar negativamente a qualidade de vida dos pacientes ${ }^{8}$ e seus cuidadores ${ }^{1}$. A pior capacidade funcional dos pacientes é fator preditor para a pior qualidade de vida dos cuidadores ${ }^{9}$.

A qualidade de vida consiste em bem-estar físico, mental, social e não somente na ausência de doenças ou enfermidades segundo a Organização Mundial da Saúde (OMS). Diz respeito a percepção do indivíduo sobre a sua posição na vida, no contexto cultural, no sistema de valores em que ele vive e em frente a seus objetivos, expectativas e preocupações. É um conceito amplo que engloba as experiências humanas, as percepções, e inclui dimensões culturais, psicológicas, físicas, espirituais, interpessoais, financeiras, políticas, fisiológicas e temporais ${ }^{10}$.

A trajetória de doenças crônicas podem ter efeitos adversos graves sobre o cuidador ${ }^{2}$ o que deixa claro a necessidade de manter o seu bem-estar e uma vida saudável para ofertar cuidados ao familiar necessitado. Dessa forma, a avaliação da qualidade de vida do cuidador torna-se relevante para que o profissional de saúde conheça suas necessidades, trace metas e programe ações minimizando os aspectos negativos do processo de cuidar ${ }^{11}$.

As publicações referentes a cuidadores de pacientes com DMD não analisam a evolução da qualidade de vida conforme a progressão da doença ${ }^{9}$. 0 cuidado prestado é estressante ${ }^{11}$ e pode afetar o cuidador conforme o paciente se torna mais dependente. A maioria dos estudos é voltada para a qualidade de vida dos pacientes com DMD, sendo restritas as publicações com cuidadores familiares.

\section{OBJETIVO}

Analisar a evolução da qualidade de vida de cuidadores de pacientes com Distrofia Muscular de Duchenne após um ano de reabilitação, segundo o grau de estadiamento da doença. 


\section{MÉTODOS}

Trata-se de um estudo do tipo quantitativo e longitudinal. Foi aprovado pelo comitê de ética em pesquisa da Pontifícia Universidade Católica de Goiás (PUC Goiás), segundo parecer 1.547.418. Foi realizado de acordo com as recomendações da Resolução 466/12 do Conselho Nacional de Saúde. Os dados foram coletados em um centro de reabilitação de Goiânia. A primeira coleta de dados ocorreu de outubro a dezembro de 2016 e a segunda de outubro a dezembro de 2017.

A amostra inicial contou com 30 cuidadores e seus respectivos pacientes. Após um ano, foram retirados do estudo três participantes, pois um paciente veio a óbito por complicações respiratórias e dois perderam contato com a instituição. Desta forma, a amostra de conveniência foi composta por 27 cuidadores familiares e seus respectivos pacientes. Foram incluídos pacientes com diagnóstico confirmado de DMD e seus cuidadores informais familiares; e que estavam em processo de reabilitação. Foram excluídos os cuidadores profissionais e pacientes com outras doenças neurológicas associadas.

Na primeira coleta, os participantes assinaram o Termo de Assentimento (para pacientes com idade inferior a 18 anos) e o Termo de Consentimento Livre e Esclarecido (para os cuidadores e pacientes com idade igual ou superior a 18 anos); os cuidadores responderam uma ficha de perfil sociodemográfico desenvolvida pelos pesquisadores (contendo informações gerais sobre os pacientes e os cuidadores) e o instrumento de Medida da Qualidade de Vida da Organização Mundial de Saúde (WHOQOL-bref); e os pacientes foram submetidos a avaliações físicas para preenchimento da Escala de Vignos.

Após um ano, os mesmos cuidadores e pacientes foram novamente abordados. Os cuidadores responderam 0 WHOQOL-bref e os pacientes foram reavaliados para preenchimento da Escala de Vignos.

Neste período de um ano, os indivíduos passaram por um processo de reabilitação multiprofissional (Fisioterapia, Terapia Ocupacional, Fonoaudiologia, Psicologia, Fisiatria, Ortopedia, Neurologia, Pneumologia, dentre outras) oferecido pela própria instituição. A frequência e o tipo de intervenção variaram de acordo com a necessidade de cada pessoa. Os pesquisadores apenas avaliaram os participantes, e não foi responsabilidade dos mesmos a condução do processo de reabilitação.

A Escala de Vignos classifica a função de 0 a 10, e quanto maior a classificação, pior é o desempenho funcional. Trata-se de um método simples e de fácil aplicação, e, em razão disto, é amplamente utilizada. As atividades funcionais são voltadas para membros inferiores, que são os mais acometidos com a evolução da doença ${ }^{12}$.

O WHOQOL-bref é um instrumento utilizado para avaliar a qualidade de vida de populações adultas. Contém 26 perguntas, das quais 24 são distribuídas em quatro domínios: físico, psicológico, relações sociais e meio-ambiente. Além disso, o instrumento apresenta duas questões gerais, sendo que uma faz referência à percepção da qualidade de 
vida e a outra à satisfação com a saúde ${ }^{13}$. Os resultados dos escores brutos de cada domínio são transformados em um escore que varia de zero a 100. Quanto mais próximo o escore médio estiver de 100, mais positiva é a percepção da qualidade de vida.

Os dados foram tabulados e analisados adotando um nível de significância de $5 \%(p \leq 0,05)$. A descrição do perfil sociodemográfico e clínico do grupo foram realizados por meio de frequência absoluta e relativa para as variáveis categóricas e estatísticas descritivas para as variáveis contínuas. O teste de Shapiro-Wilk foi aplicado para verificar se as variáveis contínuas apresentaram uma distribuição normal. A comparação dos instrumentos Escala de Vignos e WHOQOL-bref da primeira e segunda coleta foi realizada utilizando o Teste t pareado. A evolução da qualidade de vida em função da fase de estadiamento (Escala de Vignos) foi realizada pela análise da variância fatorial (ANOVA-Fatorial).

\section{RESULTADOS}

A amostra foi constituída por 27 cuidadores familiares, com média de idade de 39,44 anos $( \pm 8,40)$. A renda familiar média mensal foi de $2103,29( \pm 2427,68)$ reais. A maioria dos cuidadores é do sexo feminino $(92,60)$, possui ensino médio ou fundamental $(88,80 \%)$ e não tem atividade laboral $(70,40 \%)$. Houve maior porcentagem de pessoas que afirmam receber algum tipo de benefício social (70,40\%). A maioria possui atividade de lazer $(63,00 \%)$ e tem como meio de transporte o carro $(66,70 \%)$. Apenas $14,80 \%$ dos cuidadores fazem tratamento psicológico. Em 85,20\% dos casos, 0 cuidador relatou que recebia apoio do companheiro no cuidado do paciente com DMD.

A média de idade dos pacientes com DMD foi de 14,59 anos $( \pm 4,04)$ e 66,7\% estavam matriculados em algum tipo de escola. Os cuidadores relataram que a idade de manifestação dos sintomas da DMD variou entre de 1,0 a 7,0 anos, sendo a média de idade de $3,87( \pm 1,93)$ anos. Esses pacientes ficaram incapacitados de andar em média com 9,52 $( \pm 2,36)$ anos de idade, sendo que três ainda conseguiam deambular. A tabela 1 descreve as terapias realizadas pelos participantes do estudo na instituição de reabilitação.

Tabela 1. Reabilitação multiprofissional realizada pelos pacientes durante o período de um ano. n=27, Goiânia, GO, Brasil, 2018.

\begin{tabular}{lcc}
\hline \multicolumn{1}{c}{ Reabilitação Multiprofissional } & Frequência relativa & Frequência absoluta \\
\hline Nenhuma & 2 & 3,40 \\
Fisioterapia & 25 & 42,40 \\
Fonoaudiologia & 1 & 1,70 \\
Hidroginástica & 12 & 20,30 \\
Psicologia & 4 & 6,80 \\
Terapia ocupacional & 13 & 22,00 \\
Outros & 2 & 3,40 \\
\hline
\end{tabular}

A tabela 2 apresenta os valores da Escala de Vignos, e dos domínios e escore geral do WHOQOL-bref na primeira e segunda coleta. A Escala de Vignos apresentou piora significativa após um ano. Com relação ao WHOQOL- 
bref, o domínio meio ambiente apresentou as piores médias nas duas coletas, e o domínio físico as melhores médias.

Não houve diferença significativa nas médias dos domínios e escore geral do WHOQOL-bref após um ano.

Tabela 2. Comparação da Escala de Vignos dos pacientes e dos domínios e escore geral do WHOQOL-bref dos cuidadores entre a primeira e segunda avaliação. n=27, Goiânia, GO, Brasil, 2018.

\begin{tabular}{lccc}
\hline & \multicolumn{2}{c}{ Período (Média \pm DP) } & $P$ \\
\hline Escala de Vignos & Primeira avaliação & Segunda avaliação & \\
WHOQOL-bref & $7,07 \pm 1,47$ & $7,59 \pm 1,76$ & $<0,001^{*}$ \\
$\quad$ & & & \\
Domínio físico & $65,08 \pm 12,38$ & $65,99 \pm 15,96$ & $0,70^{* *}$ \\
Domínio psicológico & $65,13 \pm 17,52$ & $61,25 \pm 17,29$ & $0,14^{* *}$ \\
Domínio social & $64,20 \pm 20,91$ & $60,19 \pm 30,43$ & $0,14^{* *}$ \\
$\quad$ Domínio meio ambiente & $53,83 \pm 18,62$ & $53,15 \pm 20,57$ & $0,78^{* *}$ \\
Escore geral & $62,06 \pm 13,55$ & $60,15 \pm 17,97$ & $0,42^{* *}$ \\
\hline
\end{tabular}

*Teste de Wilcoxon; ${ }^{* *}$ Teste t pareado

A comparação do WHOQOL-bref segundo a fase de estadiamento da Escala de Vignos foi realizada da seguinte forma: primeiro grupo é composto por pacientes que não apresentaram evolução da doença (13 pacientes); e o segundo grupo por pacientes que evoluíram pelo menos 1 ponto na Escala de Vignos (14 pacientes). Ao comparar a qualidade de vida dos cuidadores de pacientes que mantiveram a pontuação na Escala de Vignos em relação aos que pioraram após um ano, não houve diferença significativa entre os grupos, tanto nos domínios quanto no escore geral do WHOQOL-bref.

Mas ao comparar a qualidade de vida dos cuidadores de pacientes que tinham pontuação inicial na Escala de Vignos igual ou inferior a sete em relação aos com pontuação superior a sete, observou-se piora nos domínios psicológico e social apenas no grupo de cuidadores cujos filhos eram mais graves (Vignos maior que sete) (tabela 3).

Tabela 3. Domínios e escore geral do WHOQOL-bref da primeira e segunda avaliação em função da Escala de Vignos. n=27, Goiânia, GO, Brasil, 2018.

\begin{tabular}{|c|c|c|c|c|c|c|}
\hline \multirow[b]{2}{*}{ WHOQOL-bref } & \multicolumn{2}{|c|}{$\leq 7$} & \multicolumn{4}{|c|}{$>7$} \\
\hline & $\begin{array}{c}\text { Primeira } \\
\text { avaliação }\end{array}$ & $\begin{array}{l}\text { Segunda } \\
\text { Avaliação }\end{array}$ & $p^{*}$ & $\begin{array}{l}\text { Primeira } \\
\text { avaliação }\end{array}$ & $\begin{array}{l}\text { Segunda } \\
\text { Avaliação }\end{array}$ & $p^{*}$ \\
\hline Domínio físico & $\begin{array}{c}64,10 \pm \\
11,45\end{array}$ & $\begin{array}{c}66,72 \pm \\
15,80\end{array}$ & 0,41 & $\begin{array}{c}67,40 \pm \\
14,96\end{array}$ & $\begin{array}{c}64,28 \pm \\
17,30\end{array}$ & 0,30 \\
\hline Domínio psicológico & $\begin{array}{c}61,85 \pm \\
19,10\end{array}$ & $\begin{array}{c}60,95 \pm \\
18,75\end{array}$ & 0,77 & $\begin{array}{c}72,91 \pm \\
10,19\end{array}$ & $\begin{array}{c}61,98 \pm \\
14,34\end{array}$ & $0,02^{*}$ \\
\hline Domínio Social & $\begin{array}{c}61,84 \pm \\
21,39\end{array}$ & $\begin{array}{c}62,72 \pm \\
30,47\end{array}$ & 0,89 & $\begin{array}{c}69,80 \pm \\
19,91\end{array}$ & $\begin{array}{c}54,16 \pm \\
31,51\end{array}$ & $0,04^{*}$ \\
\hline Domínio meio ambiente & $\begin{array}{c}51,65 \pm \\
17,69\end{array}$ & $\begin{array}{c}50,85 \pm \\
20,52\end{array}$ & 0,79 & $\begin{array}{c}59,00 \pm \\
20,95\end{array}$ & $\begin{array}{c}58,61 \pm \\
20,98\end{array}$ & 0,93 \\
\hline Escore geral & $\begin{array}{c}59,86 \pm \\
13,20\end{array}$ & $\begin{array}{c}60,31 \pm \\
18,48\end{array}$ & 0,88 & $\begin{array}{c}67,29 \pm \\
13,77\end{array}$ & $\begin{array}{c}59,76 \pm \\
17,92\end{array}$ & 0,06 \\
\hline
\end{tabular}




\section{DISCUSSÃO}

Os pacientes com DMD apresentaram uma baixa capacidade funcional. Como esperado após um ano, foi verificada piora significativa na Escala de Vignos $(<0,001)$. Alguns estudos transversais feitos com a Escala de Vignos para quantificar a fase de estadiamento dos pacientes verificaram pontuação na escala de 7 a 8 pontos ${ }^{14,15}$. Esses dados estão de acordo com o presente estudo, pois a amostra foi composta por um maior número de pacientes que não deambulavam, justificando tal fato.

Um estudo longitudinal que utilizou essa mesma escala para análise da fase de estadiamento encontrou resultados semelhantes, evidenciando piora progressiva do paciente em um período de dois anos. Esse mesmo estudo mostrou que períodos de seis meses são suficientes para mudança na função motora em solo. Os pacientes, que tinham média de idade de 15,1 anos, apresentaram na Escala de Vignos média inicial de 5,48 e após dois anos média de 6,136. No presente estudo, os pacientes eram mais acometidos, tinham 14,59 anos de idade e a média inicial na Escala foi de 7,07 e a final de 7,59 após o período de um ano. Portanto, a mudança na atividade motora está intrinsecamente relacionada ao envelhecimento dos pacientes e a progressão da doença. É importante salientar que todos pacientes do estudo de Ferreira et al., realizavam 1 sessão de fisioterapia aquática e 2 sessões de fisioterapia em solo por semana ${ }^{16}$. No presente estudo a reabilitação multiprofissional foi oferecida pela própria instituição e a frequência e o tipo de intervenção variaram.

Na análise da qualidade de vida dos cuidadores encontramos piores médias no domínio psicológico, social e meio ambiente na segunda coleta. Um estudo realizado em São Paulo analisou a qualidade de vida de cuidadoras/mães de filhos com DMD, Distrofia Muscular de Cinturas e Amiotrofia Espinhal Progressiva, e mostrou escores piores nos domínios psicológico, social e meio-ambiente, independentemente do tipo de distrofia avaliada ${ }^{17}$. Apesar dos resultados do presente estudo não mostrarem dados significantes, alguns autores encontraram valores médios semelhantes aos do presente estudo, mostrando que a qualidade de vida dos cuidadores de pacientes com DMD é ruim ${ }^{18,19,9,2}$.

O domínio meio ambiente apresentou as piores médias nas duas coletas. Esse domínio engloba segurança física e proteção no lar, recursos financeiros, oportunidades de adquirir novas informações e de recreação/lazer, meio de transporte e as oportunidade de trabalho. No presente estudo os recursos financeiros disponíveis no geral eram escassos. Além disso grande parte dos cuidadores não tinha atividade laboral formal, e se dedicavam aos cuidados dos paciente em tempo integral. A suspensão do trabalho é uma necessidade expressa por uma percentagem de cuidadores familiares, e está ligado ao maior impacto emocional da doença no cuidador. Isso pode ser atribuído a dois motivos principais: os pais que deixam o emprego são os que cuja doença da criança é mais grave; e desistir do trabalho estritamente significa desistir do bem-estar e satisfação pessoal, o que também leva a um contato mais contínuo e emocional com o paciente doente ${ }^{20}$. 
Ao comparar a qualidade de vida dos cuidadores em função da fase de estadiamento, mesmo os pacientes que pioraram após um ano, não houve diferença significativa, tanto nos domínios quanto no escore geral do WHOQOL-bref. Não foram encontrados estudos longitudinais para comparar os resultados, mas estudos transversais mostraram que quanto maior a idade do paciente pior a qualidade de vida do cuidador, mostrando que a evolução da doença tem um maior impacto negativo sobre a qualidade de vida do paciente e do seu cuidador ${ }^{18,19,9,2}$.

Segundo Moura et al., os cuidadores de pacientes mais velhos apresentam mais sobrecarga quando comparados com de pacientes mais novos, mostrando que o tempo de cuidado desses pacientes sobrecarrega os cuidadores e consequentemente diminui a sua qualidade de vida. Esclarece ainda que a origem da carga está relacionada especificamente com as altas demandas de cuidados ao familiar necessitado ${ }^{9}$. Ressalta-se que 0 tempo de acompanhamento dos cuidadores do presente estudo foi pequeno, o que não necessariamente demonstra um discordância dos achados dos estudos transversais.

Poucos estudos têm investigado a carga dos cuidadores de pacientes com DMD. Um estudo Brasileiro observou que a qualidade de vida ruim é explicada pela sobrecarga de cuidar de crianças com DMD. Ainda sobre esse estudo, quase $40 \%$ dos cuidadores relataram estar insatisfeitos com suas vidas ${ }^{9}$. Além disso, estresse, depressão e ansiedade têm sido descritos em grupos de cuidadores de crianças com $\mathrm{DMD}^{21}$. Alguns estudos mostram que a experiência do cuidador resulta em um fardo emocional e físico ${ }^{20,22}$.

Foi observado piora nos domínios psicológico e social dos cuidadores cujos filhos eram mais graves após um ano. $\mathrm{O}$ domínio psicológico e social englobam, sentimentos positivos e negativos, autoestima, imagem corporal e aparência, espiritualidade, relações pessoais e suporte (apoio) social. O impacto da doença na função familiar é grande em termos emocionais, e a tensão vivenciada pelos cuidadores está intrinsecamente relacionada aos pacientes. Portanto, um agravamento da qualidade de vida ou do quadro clínico das crianças está associado a um maior impacto emocional nos pais ${ }^{20}$. No presente estudo, algumas habilidades abordadas na Escala de Vignos podem ter sido determinantes para diminuir a qualidade de vida do cuidador, como inabilidade em conduzir a cadeira de rodas, não conseguir beber e comer sozinho e não sentar sem suporte na cadeira, o que consequentemente exigirá maior demanda de cuidado pelos cuidadores familiares.

Segundo Awad et al., existe maior prevalência de pequenos distúrbios psiquiátricos em cuidadores, como consequência da sua exposição ao estresse ${ }^{23}$. Uma revisão sistemática sobre intervenções para cuidadores de pacientes com doenças incuráveis e potencialmente fatais mostrou que as intervenções de apoio familiar tinham impacto positivo nos cuidadores e levou a mudanças positivas no fardo psicológico ${ }^{24}$. Diante dos resultados encontrados no presente estudo, é necessário intervenções de apoio, que podem ajudar a reduzir o sofrimento psíquico do cuidador, levando em 
consideração ainda que apenas $18,51 \%$ dos cuidadores realizavam tratamento psicológico.

Um estudo realizado na Itália mostrou que a carga psicológica é muito influenciada pelas oportunidades dos parentes de manter relacionamentos, que resulta em menor carga entre familiares que estão empregados, menos envolvidos nos cuidados diários do paciente e que mantêm contatos sociais ${ }^{25}$. Esses achados confirmam a importância das famílias compartilharem suas experiências com outras, através da participação em associações e grupos de autoajuda ${ }^{26,27}$.

\section{CONSIDERAÇÕES FINAIS}

Os cuidadores familiares de paciente com DMD apresentam comprometimento da qualidade de vida, principalmente no domínio meio-ambiente. Após um ano de reabilitação, apenas cuidadores de pacientes mais graves, que encontram-se em fase de estadiamento da doença mais avançada, tiveram piora da qualidade de vida nos domínios psicológico e social. Esses resultados demonstram que é preciso uma intensificação dos cuidados às famílias de pacientes conforme a progressão da doença. Os profissionais da saúde devem reconhecer a qualidade de vida como uma problemática de interferência nas relações dos cuidadores familiares com pacientes com DMD. Uma intervenção baseada em toda a família e não somente no paciente com DMD deve ser considerada. Este estudo apresenta-se como fonte incentivadora para o desenvolvimento de novas pesquisas, o que poderia fornecer possibilidades de desenvolvimento de estratégias de intervenção familiar.

\section{REFERÊNCIAS}

1. Bendixen RM, Senesac C, Lott DJ, Vandenborne K. Participation and quality of life in children with Duchenne muscular dystrophy using the International Classification of Functioning, Disability, and Health. Health Qual Life Outcomes. 2012;22:43-44

2. Landfeldt E, Lindgren P, Bell CF, Guglieri M, Straub V, Lochmüller H. Quantifying the burden of caregiving in Duchenne muscular dystrophy. Journal of Neurology 2016;1-10

3. Malik V, Rodino-klapac LR, Viollet L, Mendell JR. Aminoglycoside-induced mutation suppression (stop codon readthrough) as a theratpeutic strategy for Duchenne muscular dystrophy. Therapeutic Advances in Neurological Disorders, 2010;(3)6:379-389

4. Wiski MB, Souza MC. Perfil clinico e funcional da distrofia muscular de Duchenne: atuação da enfermagem no tratamento e diagnóstico precoce. Ensaios e Ciência: Ciências Biológicas, Agrárias e da Saúde, 2015(19)2:81-88

5. Carbonero FC, Zago GM, Campos D. Tecnologia assistiva na distrofia muscular de Duchenne: 
aplicabilidade e benefícios. Revista Neurociência, 2012;20(1):109-116

6. Fernandes LAY, Caromano FA, Assis SMB, Hukuda ME, Voos MC, Carvalho EV. Relationship between the climbing up and climbing down stairs domain scores on the FES-DMD, the score on the vignos scale, age and timed performance of functional activities in boys with Duchenne muscular dystrophy. Brazilian Journal of Physical Therapy, 2014;(18)6:513-520

7. Pangalila RF, Van Den Bos GAM, Bartels B, Bergen MP, Kampelmacher MJ, Stam JH, et al., Quality of life of adult men with duchenne muscular dystrophy in the netherlands: implications for care. Journal Rehabilitation Medical, 2015;(47):161-166

8. Oliveira SS, Blascovi-Assis SM, Caromano FA. Qualidade de vida de crianças com distrofia muscular: estudo de dois casos. Licere 2013;16(3):1-25

9. Moura MCDS, Wutzki HC, Voos MC, Resende MBD, Reed UC, Hasue RH. Is functional dependence of Duchenne muscular dystrophy patients determinant of the quality of life and burden of their caregivers? Arquivos Neuropsiquiatria, 2015;(73)1:52-57

10. The WHOQOL Group. The World Health Organization quality of life assessment (WHOQOL): position paper from the World Health Organization. Social Science and Medicine, 1995;(41)10:1403-1409

11. Costa TF, Gomes TM, Viana LRC, Martins KP, Costa KNFM. Acidente vascular encefálico: características do paciente e qualidade de vida de cuidadores. Revista Brasileira de Enfermagem, 2016;69(5):933-939

12. Vignos PJJr, Spencer GEJr. Archibald, K.C. Management of progressive muscular dystrophy in childhood. JAMA, 1963;(184):89-96

13. Fleck MPA, Louzada S, Xavier M, Chachamovich E, Vieira G, Santos L, Pinzon V. Application of the Portuguese version of the abbreviated instrument of quality life WHOQOL-BREF. Revista de Saúde Pública. 2000;(34):178-183

14. Opstal SL, Jansen M, Van NA, De Groot IJ. Health凸related quality of life and its relation to disease severity

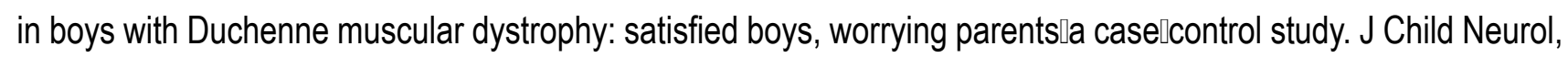
2014;(29):1486-1495

15. Sá CSC, Fagundes IK, Araújo TB, OliveiraASB, Fávero FM. The relevance of trunk evaluation in Duchenne muscular dystrophy: the segmental assessment of trunk control. Arq Neuropsiquiatr. 2016;74(10):791-5

16. Ferreira AVS, Goya PSA, Ferrari R, Durán M, Franzini RV, Coromano et al., Comparison of motor function in patients with Duchenne muscular dystrophy in physical therapy in and out of water: 2-year follow-Up. Acta Fisiátrica, 2015;(22)2:51-54

17. Simon, V. A. Qualidade de vida em crianças e adolescentes com doenças neuromusculares e validação 
de dois questionários para o português: Life Satisfaction Index for Adolescents - LSI-A e Pediatric Quality of Life Inventory Duchenne Muscular Dystrophy Module. Tese (Doutorado) - Faculdade de medicina, Universidade de São Paulo. São Paulo, 2016;229

18. Hwang JW, Koo YJ. Depressive Symptoms and Subjective Quality of Life in Parents of Boys with Duchenne/Becker Muscular Dystrophy. Journal of the Korean Academy of Child and Adolescent Psychiatry, 2010;(21)2:103-109

19. Çakaloz B, Ünlü G, Çardak GT, Kurul S. Duchenne Muskuler Distrofi ve Dikkat Eksikliği Hiperaktivite Bozukluğu Olan Çocukların Annelerinin Yaşam Kaliteleri. Pamukkale Tıp Dergisi 2010;3(2):90-95

20. Baiardini I, Minetti C, Bonifacino S, Porcu A, Klersy C, Petralia P, et al., Quality of life in Duchenne muscular dystrophy: The subjective impact on children and parents. J Child Neurol. 2011;26(6):707-713

21. Kenneson A, Bobo JK. The effect of caregiving on women in families with Duchenne/Becker muscular dystrophy. Health Soc Car Community, 2010;(18):520-528

22. Boyer F, Drame M, Morrone I, Novella JL: Factors relating to carer burden for families of persons with muscular dystrophy. J Rehabil Med 2006;38(5):309-315

23. AwadAG, Voruganti LN. The burden of schizophrenia on caregivers. Pharmacoeconomics. 2018;26(2):149162

24. Candy B, Jones L, Drake R, Leurent B, King M: Interventions for supporting informal caregivers of patients in the terminal phase of a disease. Cochrane Database Syst Rev 2011(6).

25. Larkindale J, Yang W, Hogan PF, Simon CJ, Zhang Y, Jain A, et al., Cost of illness for neuromuscular diseases in the U.S. Muscle Nerve, 2014;(49):431-438

26. Magliano L, Patalano M, Sagliocchi A, Scutifero M, Zaccaro A, D'angelo G, et al., Burden, professional support, and social network in families of children and young adults with muscular dystrophies. Muscle Nerve, 2015;(52):13-21

27. Magliano L, Fiorillo A, De Rosa C, Malangone C, Maj M. Family burden in long-term diseases: a comparative study in schizophrenia vs. physical disorders. Soc Sci Med, 2005;(61):313-322 